\title{
Correction to: A simplified equation of state for polymer melts from perturbed Yukawa hard-sphere chain
}

\author{
S. M. Hosseini ${ }^{1} \cdot$ M. M. Papari $^{2} \cdot$ J. Moghadasi ${ }^{1}$
}

Published online: 24 August 2019

(c) Springer-Verlag GmbH Germany, part of Springer Nature 2019

\section{Correction to: Polym. Bull. (2012) 69:219-228 https://doi.org/10.1007/s00289-012-0760-8}

After a careful reconsideration of Ref. [1] article, several typos appeared in writing the general formalism of PYC EoS, Eqs. 2 and 4 of Ref. [1]. Since those typos were appearing in the key equations of this paper, they would cause the significant errors in the calculation of properties of studied molten polymers using "Yukawa hardsphere chain equation of state". Regarding this, the present Erratum aims to fix this issue.

The correct formalism of perturbed Yukawa hard-sphere chain in terms of the compressibility factor is as follows:

$$
Z_{S A F T}^{H C S}=\frac{P}{\rho k_{B} T}=m \frac{1+\eta+\eta^{2}-\eta^{3}}{(1-\eta)^{3}}-(m-1) \frac{1+\eta-\eta^{2} / 2}{(1-\eta)(1-\eta / 2)}
$$

Also, some typos were appearing in writing the relevant equation to the packing fraction of Yukawa hard-spheres, i.e., Eq. 4 of Ref. [1]. Regarding this, the correct equation is:

$$
\eta=\frac{m \pi \rho \sigma^{3}}{6}
$$

The new affiliation of authors

S. M. Hosseini is Department of Chemistry, University of Hormozgan, Bandar Abbas 71961

The original article can be found online at https://doi.org/10.1007/s00289-012-0760-8.

S. M. Hosseini

seied_mostafa_hosseini@yahoo.com

1 Department of Chemistry, Shiraz University, 71454 Shiraz, Iran

2 Department of Chemistry, Shiraz University of Technology, 71555-313 Shiraz, Iran 
J. Moghadasi is Department of Chemistry, Shiraz University of Technology, 71555-313 Shiraz, Iran

\section{Reference}

1. Hosseini SM, Papari M, Moghadasi J (2012) A simplified equation of state for polymer melts from perturbed Yukawa hard-sphere chain. Polym Bull 69:219-228

Publisher's Note Springer Nature remains neutral with regard to jurisdictional claims in published maps and institutional affiliations. 